\title{
XXVI. On the difficulties of constructing a theory of the collapse of boiler-flues
}

\author{
A. B. Basset M.A. F.R.S.
}

To cite this article: A. B. Basset M.A. F.R.S. (1892) XXVI. On the difficulties of constructing a theory of the collapse of boiler-flues, Philosophical Magazine Series 5, 34:208, 221-233, DOI: 10.1080/14786449208620314

To link to this article: http://dx.doi.org/10.1080/14786449208620314

曲 Published online: 08 May 2009.

Submit your article to this journal

Џll Article views: 3

Q View related articles $₫$ 
LONDON, EDINBURGH, AND DUBLIN

\title{
PHILOSOPHICAL MAGAZINE
}

\author{
AND \\ JOURNAL OF SCIENCE.
}

[FIFTH SERIES.]

$S E P T E M B E R \quad 1892$.

XXVI. On the Difficulties of Constructing a Theory of the Collapse of Boiler-flues. By A. B. BASSET, M.A., F.R.S.*

1. T $\mathrm{T}$ has frequently happened in the history of mathe1 matics that results which are substantially correct have originally been obtained by processes which are in many cases inadequate and sometimes erroneous. When a branch of science is in its infancy, it is not surprising that many of the methods adopted by the pioneers of the subject, in attempting to discover the solutions of problems which have never been previously attacked, are subsequently found to be defective; and after fulfilling their object by contributing to the advancement of science, have ultimately to be consigned to oblivion. The theory of elastic plates and shells affords a striking illustration of these remarks; for the investigations of Clebsch and many of the earlier writers upon this subject furnish numerous results which are substantially accurate, although many of their arguments and methods are altogether unsound.

2. Clebsch and some of his followers have attempted to construct a theory of plane plates upon the hypothesis that the stresses $\mathrm{R}, \mathrm{S}, \mathrm{T}$ are ascurately zero throughout the substance of the plate, where $R$ is the normal traction, and $S$ and $T$ are the two shearing-stresses tending to produce rotation about two perpendicular lines which are parallel to the middle surface of the plate. Clebsch then proceeds to integrate the

* Communicated by the Author.

Phil. Mag. S. 5. Vol. 34. No. 208. Sept. 1892. 
general equations of an elastic solid on this hypothesis. Under any circumstances, an assumption of this character is an exceedingly objectionable one to found a theory of thin plates upon ; and Clebsch himself appears to have been conscious of the imperfections of his theory, for he says (Théorie de l'Elasticité, Saint Venant's translation, p. 296):- "Ces équations (i. e. $\mathrm{R}=\mathrm{S}=\mathrm{T}=0$ ) ne s'appliquent tout d'abord qu'aux valeurs de $z$ correspondant aux bases ou faces extrêmes de la plaque : mais j'examinerai seulement les états d'équilibre dans lesguels elles sont satisfaites en tous les points du corps." The assumption itself can, however, be shown to be erroneous; for if a plane plate of thickness $2 h$ is slightly bent into a cylinder of any form, and $\mathrm{N}$ be the normal shearing-stress across, and $G$ the flexural couple about a generator, one of the equations of equilibrium is

But

$$
\frac{d \mathrm{G}}{d s}+\mathrm{N}=0
$$

$$
\mathrm{N}=\int_{-h}^{h} \mathrm{~T} d h^{\prime}
$$

where $l^{\prime}$ is the distance of any point of the substance of the shell from the middle surface; if, therefore, $T$ were zero, $N$ would be zero and $G$ constant, which is wrong, since $G$ is known to depend upon the change of curvature. Similar observations apply to S. The fact that two of Clebsch's fundamental assumptions can thus be proved to be erroneous, vitiates every theory in which they form an essential part of the investigation.

3. But notwithstanding the defective character of the theories referred to, Clebsch's assumptions, when properly employed, are capable of furnishing a perfectly satisfactory theory of thin plates and shells, subject to the following limitations:-

(i) The surfaces of the plate or shell must not be subjected to any external pressures or tangential stresses.

(ii) The results of the theory must be regarded as approximate ones, in which powers of the thickness higher than the cube are neglected.

The reason of this is that $R, S$, and $T$ are each of the form $\alpha h^{2}+2 \beta h h^{\prime}+\gamma h^{2}+$ terms involving higher powers of $h, h^{\prime}$, where $\alpha, \beta, \gamma$ are functions of the displacements of the middle surface and their differential coefficients; whence it can be shown that if these stresses were retained, they would introduce into the expression for the energy of the system higher terms than $h^{3}$. Since such terms are to be neglected, 
the assumption that R, S, T may be treated as zero gives a perfectly correct expression for the energy to the above order of approximation.

For a fuller discussion of this assumption and the results to which it leads, the reader is referred to the following papers *.

4. When the surfaces of the shell are subjected to external pressure, the results furnished by the above hypothesis are no longer correct. This was tirst pointed out by Lord Rayleigh $\dagger$, and shortly afterwards by myself. I wrote $\ddagger$ :-

"The fundamental hypothesis that R, S, T may be treated as zero, is not true when the surfaces of the shell are subjected to external pressures or tangential stresses; for if the convex and concave surfaces of the shell are subjected to pressures $\Pi_{1}, \Pi_{2}$, the value of $R$, as we pass through the substance of the shell from its exterior to its interior surface, must vary from $-\Pi_{1}$ to $-\Pi_{2}$, and consequently (excepting in very special cases) $R$ will contain a term independent of the thickness. Hence the theory developed in the present paper is not applicable to problems relating to the collapse of boiler-flues. ... In order to obtain a theory which would enable such questions to be mathematically investigated, it would be necessary to find the values of the additional terms in the variational equation of motion which depend upon the external pressures ; and this is a problem which awaits solution."

5 . The problem of the collapse of a boiler-flue, in its simplest form, may be stated as follows :-Let the flue be regarded as indefinitely long, and be cylindrical ; let $a+h$ and $a-h$ be the external and internal radii of its surfaces; and let the outer and inner surfaces be subjected to pressures $\Pi_{1}, \Pi_{2}$. Then the condition of stability requires that a certain relation should exist between these four quantities, which may be written

$$
\mathrm{F}\left(a, h, \Pi_{1}, \Pi_{2}\right)>0 \text {. }
$$

If this condition is not satisfied, the equilibrium will be unstable, and a disturbance will cause the flue to collapse.

If the flue be regarded as of finite length $l$, a correction is required whose importance depends upon the magnitude ratio of $a$ to $l$.

Taking the simplest case of an indefinitely long tube, the problem of determining $\mathrm{F}$ may be attacked in two different ways. In the first place, let the flue be slightly deformed, and let the period of the small oscillations be found; then

- Lord Rayleigh, Proc. Lond. Math. Soc. vol. xx. p. 225. Basset, ibid. vol. xxi. pp. 33, 53; Phil. Trans. 1890, p. 433.

† Proc. Lond. Math. Soc. vol. zx. p. 373.

f Phil. Trans. 1890, p. 437 . 


\section{Mr. A. B. Basset on the Difficulties of Constructing}

the condition of stability requires, that the periods should be real quantities. In the second place, let the potential energy in the deformed state be found; then the condition of stability requires, that the potential energy of the flue when in equilibrium should be a minimum. Either of these methods will determine the mathematical form of the function $\mathrm{F}$.

In order to apply the first method, it might be supposed that we might start with the general equations of an elastic solid, and calculate the values $w_{0}, v_{0}$ of the radial and tangential displacements when the flue is in equilibrium. Having done this, let $w_{0}+w_{1}, v_{0}+v_{1}$ be the complete values of the displacements when the flue is performing small oscillations. The values of all these quantities can be obtained from the equations of motion; and by means of the boundary conditions, some of the arbitrary constants which occur in the solution can be determined, and the rest eliminated; and the resulting equation will give the period.

But in attempting to apply this method, it will be found that the pressures $\Pi_{1}, \Pi_{2}$ disappear ; consequently the periods of vibration are the same as the free periods, when the flue is not subjected to any surface-pressure. This result is obviously wrong. The reason is, that the general equations of an elastic solid in their ordinary form are linear. In order to solve the problem by the first method, it would be necessary to take account of certain quadratic terms, in which quantities upon which the motion depends enter into combination with the surface-pressures.

The problem may be illustrated by considering the propagation of waves in a liquid. When the liquid is initially at rest, all quadratic terms are to be neglected; but when the liquid possesses an independent motion, all quadratic terms which depend upon this independent motion must be retained.

The application of the second method involves a knowledge of the expression for the potential energy due to deformation. The form of this function is known when the surfaces of the flue are free from external pressures *; but this expression, as we have already pointed out, is inapplicable when there are external pressures.

6 . In order to understand more clearly the necessity of retaining the above-mentioned quadratic terms when applying the first method, let us consider the stability of a rod of length $l$ which is subjected to a tension $\mathrm{T}_{1}$. Then employing the notation and method explained in my 'Elementary

* Phil. Trans. 1890, p. 443, equation (24). 
a Theory of the Collapse of Boiler-flues.

Treatise on Hydrodynamics and Sound,' ch. viii. p. 147, we have

whence

$$
\frac{\mathrm{A}^{\prime} \mathrm{B}^{\prime}}{\mathrm{AB}}=1+\frac{\mathrm{T}_{1}}{q \omega}
$$

Accordingly

$$
\frac{\mathrm{P}^{\prime} \mathrm{Q}^{\prime}}{\mathrm{AB}}=\left(1+\frac{\mathrm{T}_{1}}{q \omega}\right)\left(1+\frac{h}{\rho^{\prime}}\right)
$$

whence

$$
\begin{aligned}
\mathrm{T}^{\prime} & =\frac{\left(q+\mathrm{T}_{1} / \omega\right)\left(1+h / \rho^{\prime}\right)}{1+h / \rho}-q, \\
& =\frac{\mathrm{T}_{1}}{\omega}+h\left(q+\frac{\mathrm{T}_{1}}{\omega}\right)\left(\frac{1}{\rho^{\prime}}-\frac{1}{\rho}\right) ;
\end{aligned}
$$

$$
\mathrm{G}=\kappa^{2} \omega\left(q+\frac{\mathrm{T}_{1}}{\omega}\right)\left(\frac{1}{\rho^{\prime}}-\frac{1}{\rho}\right) . . . .
$$

When no forces other than stresses applied to the ends of the rod act, the equations of motion- $\$ 135$, equations (7) -are

$$
\left.\begin{array}{l}
\frac{d \mathrm{~T}}{d s}-\frac{\mathrm{N}}{\rho^{\prime}}=-\sigma \omega \ddot{u}, \\
\frac{d \mathrm{~N}}{d s}+\frac{\mathrm{T}}{\rho^{\prime}}=-\sigma \omega \ddot{v} \\
\frac{d \mathrm{G}}{d s}+\mathrm{N}=\sigma \kappa^{2} \omega \ddot{\phi} .
\end{array}\right\} . . . . \cdot
$$

When the rod is naturally straight and is free from stress, $\mathrm{T}, \mathrm{N}$, and $\mathrm{G}$ are zero in equilibrium ; consequently, when the rod is performing small oscillations, these quantities will be linear functions of the displacements and their differential coefficients; accordingly $\mathrm{N} / \rho^{\prime}, \mathrm{T} / \rho^{\prime}$ are small quantities of the second order, which are to be neglected. But when the rod is subjected to a tension $T_{1}$, the value of $T$ is equal to $T_{1}+T^{\prime}$, where $\mathrm{T}^{\prime}$ depends upon the motion; accordingly the quadratic term $\mathrm{T} / \rho^{\prime}$ cannot be neglected, but must be replaced by $\mathrm{T}_{1} / \rho^{\prime}$.

Writing $-d x=d s$, and recollecting that

$$
\frac{1}{\rho^{\prime}}=-\frac{d^{2} w}{d x^{2}}, \quad \ddot{\phi}=\frac{d \ddot{v}}{d x},
$$

(1) becomes

$$
\mathrm{G}=-\kappa^{2} \omega\left(q+\frac{\mathrm{T}}{\omega}\right) \frac{d^{2} w}{d x^{2}}
$$


226 Mr. A. B. Basset on the Difficulties of Constructing

whence the last two of (2) become

$$
\begin{aligned}
& \frac{d \mathrm{~N}}{d x}+\mathrm{T}_{1} \frac{d^{2} w}{d x^{2}}=\sigma \omega \ddot{v}, \\
& \kappa^{2} \omega\left(q+\frac{\mathrm{T}_{1}}{\omega}\right) \frac{d^{3} v}{d x^{3}}+\mathrm{N}=\sigma \kappa^{2} \omega \frac{d \ddot{v}}{d x} ;
\end{aligned}
$$

whence, eliminating $\mathrm{N}$, we have

$$
\kappa^{2}\left(q+\frac{\mathrm{T}_{1}}{\omega}\right) \frac{d^{4} v}{d x^{4}}-\frac{\mathrm{T}_{1}}{\omega} \frac{d^{2} v}{d v^{2}}+\sigma \frac{d^{2} w}{d t^{2}}-\sigma \kappa^{2} \frac{d^{4} v}{d x^{2} d t^{2}}=0, .
$$

which is a well-known result.

Let $l$ be the length of the rod; then we may take

$$
v \propto \epsilon^{\iota p t+\imath m x / l},
$$

where $m$ is an integer. Substituting in (3), wo obtain

$$
\sigma\left(1+\frac{\sigma \kappa^{2} m^{2} \pi^{2}}{l^{2}}\right) p^{2}=\frac{m^{2} \pi^{2}}{l^{2}}\left\{\left(q+\frac{\mathrm{T}_{1}}{\omega}\right) \frac{\kappa^{2} m^{2} \pi^{2}}{l^{2}}+\frac{\mathrm{T}_{1}}{\omega}\right\} .
$$

When $\mathrm{T}_{\mathrm{I}}$ is positive, so that the force is a tension, $p$ is always real; but when $T_{1}$ is negative and equal to $-P$, so that the force is a pressure or thrust, $p$ will be imaginary unless

$$
\mathrm{P}<\frac{\kappa^{2} \omega q}{\kappa^{2}+l^{2} / m^{2} \pi^{2}} .
$$

The least value of the expression on the right-hand side occurs when $m=1$; the condition of stability is therefore

$$
\mathrm{P}<\frac{\kappa^{2} q \omega \pi^{2} / l^{2}}{1+\pi^{2} \kappa^{2} / l^{2}} \text {. . . . . . . }
$$

If the length of the rod is large in comparison with the radius of its cross section, the term $\pi^{2} \kappa^{2} / l^{2}$ in the denominator may be neglected, and we obtain Euler's law of thrust.

7. We must now return to the subject of boiler-flues.

This problem has recently been discussed by Mr. Bryan by means of the energy method *; but his work, as distinguished from lis result, is vitiated by the assumption that the potential energy per unit of length of the cross section due to bending is equal to

$$
\frac{1}{2} \beta\left(\delta \rho^{-1}\right)^{2}, \text { or } \frac{4 m n h^{3}}{3 a^{4}(m+n)}\left(\frac{d^{2} w}{d \phi^{2}}+w\right)^{2},
$$

which, as we have already pointed out, is only true when the surfaces of the flue are free from external pressure.

- Proc. Camb. Phil. Soc. vol, vi. p. 287. 
Mr. Bryan has also attempted to introduce the external pressures into his expression for the total potential energy due to strain, by taking into account certain terms depending upon the extension of the middle surface; but this does not help the matter, for if the pressures $\Pi_{1}, \Pi_{2}$ were suitably chosen, it would be possible to prevent the middle surface from experiencing any extension, but the expression for the potential energy would still contain terms depending on $\Pi_{1}$ and $\Pi_{2}$.

8. The necessity for introducing the external pressures may also be seen as follows.

Let the flue be regarded as infinitely long, so that the problem is one of two dimensions. Let $\sigma$ be its density, $2 h$ its thickness. Then the equations of motion are*

$$
\left.\begin{array}{l}
\frac{d \mathrm{~T}}{d s}+\frac{\mathrm{N}}{\rho}=\mu \ddot{v}, \\
\frac{d \mathrm{~N}}{d s}-\frac{\mathrm{T}}{\rho}+\Pi_{2}\left(1-\frac{h}{\rho}\right)-\Pi_{1}\left(1+\frac{h}{\rho}\right)=\mu \ddot{w}, \\
\frac{d \mathrm{G}}{d s}+\mathrm{N}=0,
\end{array}\right\} .
$$

where $\rho$ is the radius of the deformed middle surface, and $\mu=2 h \sigma$.

Now if we knew the correct expression for the total energy of the system and were to apply the Principle of Virtual Work, we should obtain (i) two equations connecting the displacements $v$ and $v$; (ii) the values of $T, N$, and $G$ in terms of $v$ and $w$. And if we were to substitute these values of $\mathrm{T}$, $N$, and $G$ in equations (6), the last one would reduce to an identity, whilst the first two would reproduce the equations of motion in terms of $v$ and $w$, which we had already obtained. Since the second of (6) contains the pressures $\Pi_{1}, \Pi_{2}$, it follows that the expression for the energy must also contain these quantities.

From (6) it appears that the problem of the collapse of a boiler-flue can be solved, provided we can obtain the value of the flexural couple $G$, and provided also we assume that the extensibility of the middle surface may be neglected.

9. The expression for this couple in the case of a strained cylindrical shell is given in my paper in the 'Philosophical

* If the extensibility of the middle surface is taken into account, the right-hand sides will contain certain additional terms-see Pbil. Trans. $1890, p .439$, equations (11); but the existence of such terms does not affect my argument. 
228 Mr. A. B. Basset on the Difficulties of Constructing

Transactions'; but the surfaces of the shell are expressly assumed to be free from external pressures and tangential stresses, although the extension of the middle surface is taken into account. As the analysis in the general case of threedimensional motion is somewhat long and complicated, it will be desirable to reproduce in a concise form so much of the original work as is necessary to obtain the value of the flexural couple when the motion is supposed to be in two dimensions. We shall neglect the extension of the middle surface, but shall suppose that the shell is subjected to external pressure.

Let $v^{\prime}, w^{\prime}$ denote the tangential and normal displacements of any point of the substance of the shell; let $\sigma_{2}^{\prime}, \sigma_{3}^{\prime}$ be the extensions in these directions, and $\sigma^{\prime}$ the shearing stress. Also let the unaccented letters denote the values of the quantities at the middle surface, where $r=a$; and let the values at the middle surface of the differential coefficients of the various quantities with respect to $r$ be distinguished by brackets.

Let $r=a+h^{\prime}$, and let $2 h$ be the thickness of the shell. Then, employing Thomson and Tait's notation for stresses and elastic constants, we have

$$
\left.\begin{array}{rl}
\sigma_{2}^{\prime} & =\frac{1}{r}\left(\frac{d v^{\prime}}{d \phi}+w^{\prime}\right), \\
\sigma_{3}^{\prime} & =\frac{d w^{\prime}}{d r}, \\
w^{\prime} & =\frac{d v^{\prime}}{d r}-\frac{v^{\prime}}{r}+\frac{1}{r} \frac{d w^{\prime}}{d \phi} .
\end{array}\right\} . . . . .
$$

Now

$$
\begin{aligned}
\mathrm{R}^{\prime} & =(m+n) \sigma_{3}+(m-n) \sigma_{2}^{\prime} \\
& =(m+n) \sigma_{3}+\left\{(m+n)\left(\frac{d \sigma_{3}}{d r}\right)+(m-n)\left(\frac{d \sigma_{2}}{d r}\right)\right\} h^{\prime}+\ldots,
\end{aligned}
$$

for since the middle surface is supposed to be inextensible, $\sigma_{2}=0$.

Since $\mathbf{R}^{\prime}$ is some function of $h$ and $h^{\prime}$, it follows from Taylor's theorem that

$$
\mathrm{R}^{\prime}=\mathrm{A}+\mathrm{A}_{1} h^{\prime}+\mathrm{A}_{2} h_{2}^{\prime}+\ldots . .
$$

where $A, A_{1} \ldots$ are unknown functions of the displacements and the thickness. Accordingly, equating coefficients and putting

$$
\mathrm{E}=(m-n) /(m+n), . \quad \text {. . . . }
$$


we get

$$
\left.\begin{array}{c}
\sigma_{3}=\mathrm{A} /(m+n) \\
\left(\frac{d \sigma_{3}}{d r}\right)=\frac{\mathrm{A}_{1}}{m+n}-\mathrm{E}\left(\frac{d \sigma_{2}}{d r}\right)
\end{array}\right\}
$$

From (7) we get

$$
\begin{aligned}
\left(\frac{d \sigma_{2}}{d r}\right) & =\frac{1}{a}\left\{\left(\frac{d^{2} v}{d r d \phi}\right)+\sigma_{3}\right\} \\
& =\frac{1}{a}\left\{\frac{d \varpi}{d \phi}+\frac{\mathrm{A}}{m+n}-\frac{w}{a}-\frac{1}{a} \frac{d^{2} w}{d \phi^{2}}\right\} .
\end{aligned}
$$

If $G$ be the flexural couple about a generator, measured in the direction in which the curvature diminishes,

But

$$
\mathrm{G}=-\int_{-h^{\prime}}^{h} \mathrm{Q}^{\prime} h^{\prime} d h^{\prime}
$$

$$
\begin{aligned}
\mathrm{Q}^{\prime} & =(m+n) \sigma_{2}^{\prime}+(m-n) \sigma_{3}^{\prime} \\
& =(m-n) \sigma_{3}+\left\{(m+n)\left(\frac{d \sigma_{2}}{d r}\right)+(m-n)\left(\frac{d \sigma_{3}}{d r}\right)\right\} h^{\prime} \\
& =\mathrm{EA}+\left[\mathrm{EA}_{1}+\frac{4 m n}{(m+n) a}\left(\frac{d \varpi}{d \phi}+\frac{\mathrm{A}}{m+n}-\frac{w}{a}-\frac{1}{a} \frac{d^{2} w}{d \phi^{\prime}}\right)\right] h^{\prime} ;
\end{aligned}
$$

accordingly,

$$
\mathrm{G}=-\frac{2}{3} \mathrm{EA}_{1} h^{3}-\frac{8 m n h^{3}}{3(m+n) a}\left(\frac{d \sigma}{d \phi}+\frac{\mathrm{A}}{m+n}-\frac{w}{a}-\frac{1}{a} \frac{d^{2} w}{d \phi^{2}}\right) .
$$

Now $\approx h^{3}$ may be neglected when the surfaces of the shell are free from tangential stresses; and $A_{1} h^{3}$ and $A h^{3}$ may be neglected when the surfaces are free from normal stresses or pressures. Under these circumstances we obtain

$$
\mathrm{G}=\frac{8 m n l^{3}}{3(m+n) a^{2}}\left(\frac{d^{2} w}{d \phi^{2}}+w\right), . \quad . \quad .
$$

which is the well known result in this case, and shows that the flexural couple is proportional to the change of curvature. But when the surfaces of the shell are subjected to external and internal pressures $\Pi_{1}, \Pi_{2}$, the terms involving $A, A_{1}$ cannot be assumed to be negligible when multiplied by $h^{3}$. The quantity $A$ is the value of $R$ at the middle surface of the shell; and since $R$ varies from $-\Pi_{2}$ to $-\Pi_{1}$, as we pass from the interior to the exterior of the shell, it is evident that A must have some value intermediate between these two quantities. It therefore follows that under these circumstances the expression (14) is erroneous; the correct 
expression is given by (13), which contains the three unknown quantities $\mathrm{A}, \mathrm{A}_{1}$, and $\varpi$.

10. Although the existence of external pressures renders it inadmissible to treat $R$ as zero, yet the stresses $S$ and $T$ still vanish at the surfaces of the shell. I have shown in my papers that the terms of lowest order which these stresses contain are quadratic functions of $h, h^{\prime}$; and the arguments by which this result was obtained are unaffected by the existence of external pressures. Since the problem we are discussing is one of two dimensions, $\mathrm{T}$ will be accurately zero; also since

$$
\mathrm{N}=\int_{-h}^{h} \mathrm{~S}^{\prime} d h^{\prime}
$$

and $\mathrm{S}^{\prime}$ is a quadratic function of $h$ and $h^{\prime}$, it follows that $\mathrm{N}$ must be proportional to $h^{3}$, whence by the third of $(6)$, $d G / d s$ is proportional to $h^{3}$.

11. We have now to consider how $\mathrm{A}$ and $\mathrm{A}_{1}$ depend upon $h$.

Since the quantity $A$ is the value of $R$ at the middle surface of the shell, it cannot contain any negative powers of $h$, otherwise $\mathrm{R}$ would increase indefinitely as the thickness of the shell diminishes indefinitely, which is impossible, since $R$ must lie between $-\Pi_{1}$ and $-\Pi_{2}$. It is, however, possible for A to contain a term independent of the displacements; but such a term need not be considered, inasmuch as it disappears on differentiation.

The quantity $A_{1}$ might, however, contain a term involving $h^{-1}$; for the value of $R$ at a point near the middle surface is

$$
\mathrm{A}+\mathrm{A}_{1} h^{\prime}+\ldots
$$

When the thickness of the shell diminishes indefinitely, $h^{\prime}$ approaches the limit $h$, but in such a manner that $h^{\prime}$ is always less than $h$; if therefore $A_{1}$ contained a term involving $h^{-1}$, the quantity $A_{1} h^{\prime}$ would not become infinite in the limit. On the other hand, if $A_{1}$ did contain such a term it would necessarily be independent of the displacements, inasmuch as the term in question must disappear on differentiation, otherwise $d \mathrm{G} / d s$ would not be proportional to $h^{3}$.

It therefore follows that the only portions of $A, A_{1}$ which it is necessary to consider are those portions which are independent of $h$. I regret to say that I have not succeeded in discovering any method by which their values may bo rigorously deduced; but although as a rule I distrust arguments founded on general reasoning, yet the difficulty of obtaining the values of these quantities by a rigorous mathematical investigation having hitherto proved insuperable, 
renders it necessary in the present case to have recourse to such arguments.

12. When a flexural couple is applied to a thin shell, it produces extension of the middle surface and change of curvature. Under these circumstances the most natural hypothesis to make is, that this couple is a linear function of the extensions and the changes of curvature, together with a constant term depending on the external pressures. When there are no external pressures this hypothesis may be proved to be true by the method of my former paper; but when the shell is subjected to such pressures, every circle whose plane is perpendicular to the axis of the cylinder is elongated or contracted, independently of the strain produced by the couple. Accordingly we should anticipate that the coefficients of the extensions and the changes of curvature would contain terms depending on the pressures; and we shall therefore assume that

$$
\begin{aligned}
\mathrm{G}= & \frac{8 h^{3}}{3 a^{3}}\left(\frac{m n}{m+n}+\alpha\right)\left(\frac{d^{2} w}{d \phi^{2}}+w\right) \\
& +\frac{4 h^{3}}{3 a^{2}}\left\{\frac{m n(m-n)}{(m+n)^{2}}+\beta\right\}\left(\frac{d v}{d \phi}+w\right)+\gamma, .
\end{aligned}
$$

where $\alpha, \beta, \gamma$ are quantities which depend on the pressures.

When there are no external pressures, $\alpha, \beta, \gamma$ are each zero, and the above expression for $G$ reduces to that given in my former paper-see Phil. Trans. 1890, p. 441, equations (14) and (16). It will be noticed that this hypothesis is consistent with (13), as it involves nothing more than the assumption of certain definite values for the undetermined quantities $A, A_{1}$.

The preceding argument though plausible is not entirely free from danger. When a thin shell is free from surface forces, it might be argued that the effect of any stress is to produce extension, change of curvature, and torsion; and that consequently the expression for the potential energy must be a quadratic function of the quantities by which these three states are specified. If, however, the expressions for the potential energy of a thin cylindrical or spherical shell be examinedPhil. Trans. 1890 , p. 443, equation (24), and p. 467, equation (16) -it will be found that they contain certain terms depending on the differential coefficients of the extensions.

13. One further difficulty still remains. The equations (6) for determining the small oscillations of the flue involve the two displacements $v$ and $w$, and the stresses $\mathrm{T}, \mathrm{N}, \mathrm{G}$. By means of the above value of $G$ these stresses can be eliminated, 


\section{Mr. A. B. Basset on the Difficulties of Constructing}

and a single equation will remain connecting $v$ and $w$. This is insufficient to determine the period, and the only course open to us is to assume that the middle surface is inextensible, which gives a further relation between $v$ and $w$, and furnishes a sufficient number of equations. I am inclined to think that the neglect of the extension of the middle surface is not likely to lead to any serious error in the final result; but at the same time the solution cannot be regarded as perfectly rigorous, for when the surfaces are subjected to uniform pressure (which is the case we are considering), an extension of the middle surface must necessarily take place unless the pressures are specially adjusted.

14. We are now in a position to obtain a solution of the problem of the collapse of a boiler flue, which though imperfect, is probably substantially correct.

When the natural form of the cylinder is circular, $\mathrm{N}$ is zero in equilibrium; accordingly we may write $\mathrm{N} / \rho=\mathrm{N} / a$. Also if the extension of the middle surface be neglected,

$$
\left.\begin{array}{l}
d s=a d \phi, \\
\frac{1}{\rho}=\frac{1}{a}-\frac{1}{a^{2}}\left(\frac{d^{2} w}{d \phi^{2}}+w\right), \\
\frac{d v}{d \phi}+w=0 .
\end{array}\right\} . . . .
$$

The equations of motion ( 6 ) now become

$$
\begin{aligned}
& \frac{d \mathrm{~T}}{d \phi}+\mathrm{N}=\mu a v, \\
& \frac{d \mathrm{~N}}{d \phi}-\mathrm{T}+\left(\Pi_{2}-\Pi_{1}\right) a-\left(\Pi_{2}+\Pi_{1}\right) h+\left(\Pi_{2}-\Pi_{1}\right)\left(\frac{d^{2} w}{d \phi^{2}}+w\right)=\mu a \ddot{w}, \\
& \frac{d \mathrm{G}}{d \phi}+\mathrm{N} a=0 .
\end{aligned}
$$

From the first two we get

$$
\frac{d^{2} \mathrm{~N}}{d \phi^{2}}+\mathrm{N}+\left(\Pi_{2}-\Pi_{1}\right) \frac{d}{d \phi}\left(\frac{d^{2} w}{d \phi^{2}}+w\right)=\mu a\left(\frac{d \ddot{w}}{d \phi}+\ddot{v}\right) .
$$

Let $\mathrm{D}=d / d \phi$; then, since the extension of the middle surface is neglected, we shall have

where

$$
\mathrm{G}=\mathrm{I}\left(\mathrm{D}^{2}+1\right) w, \text {. . . . . }
$$

$$
\mathrm{I}=\frac{8 h^{3}}{3 a^{2}}\left(\frac{m n}{m+n}+\alpha\right) \ldots . . .
$$


a Theory of the Collapse of Boiler-fues.

Accordingly from (18), (19), (20), and the last of (16) and (17) we get

$$
\left\{\mathrm{I} a^{-1}\left(\mathrm{D}^{2}+1\right)+\left(\Pi_{1}-\Pi_{2}\right)\right\}\left(\mathrm{D}^{2}+1\right) \mathrm{D}^{2} w+\mu a\left(\mathrm{D}^{2}-1\right) \ddot{w}=0 .
$$

To solve this assume

$$
v \propto \epsilon^{\iota p t+i s \emptyset}
$$

where $s=2,3.4 \ldots$, then

$$
\left\{\mathrm{I} a^{-1}\left(s^{2}-1\right)-\left(\Pi_{1}-\Pi_{2}\right)\right\}\left(s^{2}-1\right) s^{2}=\mu a\left(s^{2}+1\right) p^{2} ;
$$

and therefore $p$ will be real provided

$$
\Pi_{1}-\Pi_{2}<\mathrm{I}\left(s^{2}-1\right) / a \text {. }
$$

The least value of the right-hand side occurs when $s=2$, in which case

$$
\begin{aligned}
\Pi_{1}- & \Pi_{2}<3 \mathrm{I} / a \\
& <\frac{8 h^{3}}{a^{3}}\left(\frac{m n}{m+n}+\alpha\right) . . . . .
\end{aligned}
$$

This is the condition for the stability of the flue.

In the particular case considered by $\mathbf{M r}$. Bryan, $\mathbf{I}_{2}=0$, and since it is practically certain that $\alpha$ is a linear function of $\Pi_{1}$, we may write $\alpha=k \Pi_{1}$, where $k$ is some constant which is independent of $h$. Whence (22) becomes

$$
\Pi_{1}\left(1-\frac{8 h^{3} k}{a^{3}}\right)<\frac{8 h^{3} m n}{a^{3}(m+n)} .
$$

Since powers of $h$ higher than the cube are to be neglected, the condition of stability becomes

$$
\Pi_{1}<\frac{8 h^{3} m n}{a^{3}(m+n)} .
$$

which is Mr. Bryan's result.

Although I am disposed to think that this result is rigorously true as a first, and probably for practical purposes as a sufficient approximation, yet a rigorous mathematical theory requires a knowledge of the correct expression for the energy. An inductive process, by means of which a function is shown to satisfy all the requisite conditions, would probably be the simplest method of discovering its value.

August 9, 1892. 